\title{
On the continuity of the solutions to the Navier-Stokes equations with initial data in critical Besov spaces
}

\author{
Reinhard Farwig ${ }^{1}$ Yoshikazu Giga ${ }^{2}$ Pen-Yuan Hsu²
}

Received: 5 December 2016 / Accepted: 16 January 2019 / Published online: 7 February 2019 (c) Fondazione Annali di Matematica Pura ed Applicata and Springer-Verlag GmbH Germany, part of Springer Nature 2019

\begin{abstract}
It is well known that there exists a unique local-in-time strong solution $u$ of the initial boundary value problem for the Navier-Stokes system in a three-dimensional smooth bounded domain when the initial velocity $u_{0}$ belongs to critical Besov spaces. A typical space is $B=B_{q, s}^{-1+3 / q}$ with $3<q<\infty, 2<s<\infty$ satisfying $2 / s+3 / q \leq 1$ or $B=\stackrel{\circ}{B}_{q, \infty}^{-1+3 / q}$. In this paper, we show that the solution $u$ is continuous in time up to initial time with values in $B$. Moreover, the solution map $u_{0} \mapsto u$ is locally Lipschitz from $B$ to $C([0, T] ; B)$. This implies that in the range $3<q<\infty, 2<s \leq \infty$ with $3 / q+2 / s \leq 1$ the problem is well posed which is in strong contrast to norm inflation phenomena in the space $B_{\infty, s}^{-1}, 1 \leq s<\infty$ proved in the last years for the whole space case.
\end{abstract}

Keywords Nonstationary Navier-Stokes system · Initial values · Weighted Serrin condition $\cdot$ Limiting type of Besov space $\cdot$ Continuity of solutions $\cdot$ Stability of solutions

Mathematics Subject Classification 35Q30 · 76D05

\section{Introduction}

We consider the initial boundary value problem of the Navier-Stokes equations in a bounded domain $\Omega \subset \mathbb{R}^{3}$ with $C^{2,1}$ boundary $\partial \Omega$,

$$
\begin{gathered}
u_{t}-\Delta u+u \cdot \nabla u+\nabla p=f, \quad \operatorname{div} u=0 \quad \text { in } \quad(0, T) \times \Omega \\
\left.u\right|_{\partial \Omega}=0, \quad u(0)=u_{0},
\end{gathered}
$$

Reinhard Farwig

farwig@mathematik.tu-darmstadt.de

Yoshikazu Giga

labgiga@ms.u-tokyo.ac.jp

Pen-Yuan Hsu

pyhsu@ms.u-tokyo.ac.jp

1 Fachbereich Mathematik, Technische Universität Darmstadt, 64289 Darmstadt, Germany

2 Graduate School of Mathematical Sciences, University of Tokyo, 3-8-1 Komaba, Meguro-ku, Tokyo 153-8914, Japan 
where $T \in(0, \infty]$. We are interested in local-in-time strong solutions in a Bochner space $L^{s}\left(0, T ; L^{q}(\Omega)\right)$ or, more generally, a weighted Bochner space with weight in time,

$$
L_{\alpha}^{s}\left(L^{q}\right):=L_{\alpha}^{s}\left(0, T ; L^{q}(\Omega)\right)=\left\{v \text { measurable in }(0, T) \times \Omega:\|v\|_{L_{\alpha}^{s}\left(L^{q}\right)}<\infty\right\}
$$

with

$$
\|v\|_{L_{\alpha}^{s}\left(L^{q}\right)}:=\left(\int_{0}^{T}\left(\tau^{\alpha}\|v(\tau)\|_{q}\right)^{s} \mathrm{~d} \tau\right)^{1 / s},
$$

where $\alpha \geq 0$ and $1 \leq s<\infty$; for $s=\infty$, the standard modification for the norm $\|\cdot\|_{L_{\alpha}^{\infty}\left(L^{q}\right)}$ is to be used. By definition, $L_{0}^{s}\left(L^{q}\right)=L^{s}\left(L^{q}\right)=L^{s}\left(0, T ; L^{q}(\Omega)\right)$.

There is a large literature on the existence of a local-in-time strong solution under various regularity condition on the initial data and the external force $f[2,13-15,17,18,21,25-27]$. The first contribution in this direction seems to be the work of Kiselev and Ladyzhenskaya [19]. Since then, the condition on initial data and the external force $f$ has been weakened; in other words, $u_{0}$ can be taken in a larger space.

In the scale of Besov spaces, it is shown in [11,12] that a necessary and sufficient condition to get $L^{s}\left(L^{q}\right)$-strong solutions is that the initial data $u_{0}$ belong to a solenoidal Besov space $\mathbb{B}_{q, s}^{-1+3 / q}(\Omega)$ provided that $s=s_{q}$ where $2 / s_{q}+3 / q=1(3<q<\infty)$. In this case, the so-called Serrin class $L^{s}\left(L^{q}\right)$ allows to prove regularity and uniqueness of weak solutions of the Navier-Stokes system. See also [7] for a review.

The existence of strong solutions is extended for $s$ larger than $s_{q}$ by introducing a weighted Bochner space. In fact, in [8] a local-in-time strong solution in $L_{\alpha}^{s}\left(L^{q}\right)$ is constructed if the initial data belong to $\mathbb{B}_{q, s}^{-1+3 / q}$ for $3<q<\infty, s_{q} \leq s<\infty$ with $2 / s_{q}+3 / q=1$ and $2 / s+3 / q=1-2 \alpha$. In [9], this result is extended to the case $s=\infty$ by replacing $\mathbb{B}_{q, s}^{-1+3 / q}$ by $\stackrel{\circ}{B}_{q, \infty}^{-1+3 / q}$ which is obtained as a continuous interpolation space. In [8,9], $u_{0}$ is assumed to belong also to the space $L_{\sigma}^{2}$ to compare with weak solutions. However, just for existence of a strong solution, this additional $L_{\sigma}^{2}$ assumption is unnecessary to get an $L_{\alpha}^{s}\left(L^{q}\right)$-solution. The explanation of the Besov spaces will be given in appendix for the reader's convenience.

In this paper, we shall prove that $L_{\alpha}^{s}\left(L^{q}\right)$-solutions are indeed in $C\left([0, T] ; \mathbb{B}_{q, s}^{-1+3 / q}\right)$ for initial data $u_{0} \in \mathbb{B}_{q, s}^{-1+3 / q}$ when $s_{q} \leq s<\infty$, or in $C\left([0, T] ; \stackrel{\circ}{\mathbb{B}}_{q, \infty}^{-1+3 / q}\right)$ for $u_{0} \in$ $\stackrel{\circ}{B}_{q, \infty}^{-1+3 / q}$ when $s=\infty$; see Theorems 1.1 and 1.2 , respectively. Moreover, we will show in Theorems $1.3\left(s_{q} \leq s<\infty\right)$ and $1.4(s=\infty)$ that they are globally well posed for small initial data. Theorems 1.1 and 1.2 are in strong contrast to the so-called norm inflation phenomenon in limiting - homogeneous or inhomogeneous - Besov spaces for the corresponding Cauchy problem on $\mathbb{R}^{n}, n \geq 2$. Bourgain and Pavlovič [4] construct for any $\delta>0$ mild solutions with initial values $u_{0}$ in the Schwartz class such that $\left\|u_{0}\right\|_{\dot{B}_{\infty, \infty}^{-1}} \leq \delta$, but $\|u(t)\|_{\dot{B}_{\infty, \infty}^{-1}}>1 / \delta$ for some $0<t<\delta$. Note that on the one hand, $\dot{B}_{\infty, \infty}^{-1}$ is the largest scale-invariant Banach space of tempered distributions; see Meyer [24]. On the other hand, $B M O^{-1} \subset \dot{B}_{\infty, \infty}^{-1}$ is the largest scale-invariant space for which global well-posedness for small initial data in $B M O^{-1}$ has been proved so far, $c f$. Koch and Tataru [20]. Yoneda [32] clarifies the approach in [4] and extends the result to $\dot{B}_{\infty, s}^{-1}, s>2$, to be more precise, to a space $V$ satisfying $\dot{B}_{\infty, 2}^{-1} \subset V \subset \dot{B}_{\infty, s}^{-1}$. Wang [31] proves this norm inflation phenomenon even for all $1 \leq s<\infty$. Finally, Cheskidov and Shvidkoy [5] consider weak solutions of Leray-Hopf type such that $\lim \sup _{t \rightarrow 0}\left\|u(t)-u_{0}\right\|_{B_{\infty, \infty}^{-1}} \geq \delta_{0}$ for some $\delta_{0}>0$ independent of $u_{0}$. Since $B_{q, \infty}^{-1+3 / q}, 1<q<\infty$, is continuously embedded into $B_{\infty, \infty}^{-1}$ on $\mathbb{R}^{3}$, this result also yields the ill-posedness of weak solutions at $t=0$ measured in the space $B_{q, \infty}^{-1+3 / q}$. This negative 
result underlines the importance of using the continuous interpolation space $\mathbb{B}_{q, \infty}^{-1+3 / q}$ rather than $\mathbb{B}_{q, \infty}^{-1+3 / q}$ in Theorem 1.2.

Theorem 1.1 Let $\Omega \subset \mathbb{R}^{3}$ be a bounded domain with $C^{2,1}$ boundary. Let $0<T \leq \infty$, $2<s<\infty, 3<q<\infty$ and $0 \leq \alpha<1 / 2$ satisfy $2 / s+3 / q=1-2 \alpha$. Moreover, let $u$ be an $L_{\alpha}^{s}\left(L^{q}\right)$-strong solution with initial data $u_{0} \in \mathbb{B}_{q, s}^{-1+3 / q}$ and $f=\operatorname{div} F$ satisfying $F \in L_{2 \alpha}^{s / 2}\left(0, T ; L^{q / 2}(\Omega)\right)$. Then

$$
u \in C\left([0, T] ; \mathbb{B}_{q, s}^{-1+3 / q}\right) .
$$

Theorem $1.2(s=\infty)$ Let $\Omega, T, q$ be as in Theorem 1.1 , and let $2 \alpha=1-3 / q$. Then an $L_{\alpha}^{\infty}\left(L^{q}\right)$-strong solution $u$ with initial data $u_{0} \in \mathbb{B}_{q, \infty}^{-1+3 / q}$ and $f=\operatorname{div} F$ satisfying $F \in L_{2 \alpha}^{\infty}\left([0, T] ; L^{q / 2}(\Omega)\right)$ and $\|F\|_{L_{2 \alpha}^{\infty}\left(0, t ; L^{q / 2}\right)} \rightarrow 0$ as $t \downarrow 0$ satisfies

$$
u \in C\left([0, T] ; \stackrel{\circ}{\mathbb{B}}_{q, \infty}^{-1+3 / q}\right) .
$$

We further observe the continuity of solutions with respect to initial data and external forces.

Theorem 1.3 Under the assumptions of Theorem 1.1, let $v$ be an $L_{\alpha}^{s}\left(L^{q}\right)$-strong solution with initial data $v_{0} \in \mathbb{B}_{q, s}^{-1+3 / q}$ and external force $G \in L_{2 \alpha}^{s / 2}\left(0, T ; L^{q / 2}(\Omega)\right)$. Then there are constants $\varepsilon_{*}$ and $C$ depending only on $\Omega$ such that if $T_{0} \leq T$ is taken so that $\|u\|_{L_{\alpha}^{s}\left(0, T_{0} ; L^{q}\right)} \leq$ $\varepsilon_{*},\|v\|_{L_{\alpha}^{s}\left(0, T_{0} ; L^{q}\right)} \leq \varepsilon_{*}$, then for all $t \in\left(0, T_{0}\right)$

$$
\|(u-v)(t)\|_{\mathbb{B}_{q, s}^{-1+3 / q}} \leq C\left(\left\|u_{0}-v_{0}\right\|_{\mathbb{B}_{q, s}^{-1+3 / q}}+\|F-G\|_{L_{2 \alpha}^{s / 2}\left(0, T_{0} ; L^{q / 2}\right)}\right) .
$$

Theorem $1.4(s=\infty)$ Under the assumptions of Theorem 1.2, let $v$ be an $L_{\alpha}^{\infty}\left(L^{q}\right)$-strong solution with initial data $v_{0} \in \stackrel{\circ}{B}_{q, \infty}^{-1+3 / q}$ and external force $G \in L_{2 \alpha}^{\infty}\left(0, T ; L^{q / 2}(\Omega)\right)$ such that $\|G\|_{L_{2 \alpha}^{\infty}\left(0, t ; L^{q / 2}\right)} \rightarrow 0$ as $t \downarrow 0$. Then there are constants $\varepsilon_{*}$ and $C$ depending only on $\Omega$ such that if $T_{0} \leq T$ is taken so that $\|u\|_{L_{\alpha}^{\infty}\left(0, T_{0} ; L^{q}\right)} \leq \varepsilon_{*},\|v\|_{L_{\alpha}^{\infty}\left(0, T_{0} ; L^{q}\right)} \leq \varepsilon_{*}$, then

$$
\|(u-v)(t)\|_{\mathbb{B}_{q, \infty}^{-1+3 / q}} \leq C\left(\left\|u_{0}-v_{0}\right\|_{\mathbb{B}_{q, \infty}^{-1+3 / q}}+\|F-G\|_{L_{2 \alpha}^{\infty}\left(0, T_{0} ; L^{q / 2}\right)}\right), \quad t \in\left(0, T_{0}\right) .
$$

Theorems 1.1 and 1.2 are proved directly avoiding deep tools from interpolation theory and interpolation-extrapolation scales. The terminology of Besov spaces is only used in the statements of the theorems and in appendix where it is shown that Besov spaces are behind the norm estimates in Sects. 2, 3 and 4; see (1.6). This context is well known from the optimal spaces of initial values for a classical parabolic equation like the heat equation where $u_{0} \in B_{q, s_{q}}^{1+3 / q}=B_{q, s_{q}}^{2-2 / s_{q}}=\left(L^{q}, \mathcal{D}(\Delta)\right)_{1-1 / s_{q}, s_{q}}$ allows for a strong solution $u$ in the maximal regularity class $L^{s_{q}}(0, \infty ; \mathcal{D}(\Delta))$ over $L^{q}$. In this article, working in scale-invariant function spaces close to Serrin's class $L^{s_{q}}\left(0, T ; L_{\sigma}^{q}\right)$, the initial values are chosen from Besov spaces of solenoidal vector fields, $\mathbb{B}_{q, s}^{-1+3 / q}$ and $\stackrel{\circ}{B}_{q, \infty}^{-1+3 / q}$.

Let us recall the Helmholtz projection $\mathbb{P}_{q}: L^{q}(\Omega) \rightarrow L_{\sigma}^{q}(\Omega)$ and the Stokes operator $A=A_{q}=-\mathbb{P}_{q} \Delta$ in $L_{\sigma}^{q}(\Omega)$, the closure of $C_{c, \sigma}^{\infty}(\Omega)$ in $L^{q}(\Omega)$; here, $C_{c, \sigma}^{\infty}(\Omega)$ denotes the space of smooth solenoidal vector fields with compact support. The semigroup generated by $-A_{q}$ is denoted by $e^{-t A_{q}}$ and defines the solution operator $u_{0} \mapsto u(t)$ for the Stokes equations in case that $f=0$. Then, if $2 / s+3 / q=1-2 \alpha$, 


$$
u_{0} \in \mathbb{B}_{q, s}^{-1+3 / q} \quad \text { iff } \quad \int_{0}^{T}\left(\tau^{\alpha}\left\|e^{-\tau A} u_{0}\right\|_{q}\right)^{s} \mathrm{~d} \tau<\infty
$$

with the usual modification if $s=\infty$; for more details, see Appendix in Sect. 5. The results on continuity and well-posedness hold for a (mild) solution $u \in L_{\alpha}^{s}\left(L^{q}\right)$ of the corresponding integral equation

$$
u(t)=e^{-t A} u_{0}-\int_{0}^{t} e^{-(t-\tau) A}(\mathbb{P} \operatorname{div}(u \otimes u)-\mathbb{P} \operatorname{div} F)(\tau) \mathrm{d} \tau .
$$

In Sect. 2, we prepare abstract lemmata for Theorems 1.1-1.4 to be proved in Sect. 4. The essential technical estimates will be performed in Sect. 3. In appendix, the abstract interpolation spaces introduced in Sect. 2 are identified with solenoidal Besov spaces; these results are taken from papers by Amann [2,3] and his monograph [1].

Note that $L_{\alpha}^{s}\left(L^{q}\right)$-strong solutions in [9] are defined as the subset of classical weak solutions of Leray-Hopf type in which $u \in L_{\alpha}^{s}\left(L^{q}\right)$. Finally, related results can be found in articles by Amann [2] and Haak and Kunstmann [16] as special cases of a more general abstract theory using interpolation-extrapolation scales of Banach spaces; see Remark 4.1 for more details.

\section{Abstract spaces}

Let $X$ be a Banach space equipped with the norm $\|\cdot\|_{X}$, and let $-A$ denote the generator of a $C_{0}$-analytic semigroup $e^{-t A}$ in $X$. Assume that $\{z \in \mathbb{C}: \operatorname{Re} z \geq 0\}$ is included in the resolvent set of $A$. Then $A^{-1}: X \rightarrow \mathcal{D}(A)$ is bounded and $A: \mathcal{D}(\bar{A}) \rightarrow X$ is an isometry when $\mathcal{D}(A)$ is equipped with the homogeneous graph norm $\|A \cdot\|_{X}$. Moreover, the semigroup $e^{-t A}$ decays exponentially in time, i.e. $\left\|e^{-t A}\right\|_{\mathrm{op}(X)} \leq C_{0} e^{-v t}$ with some positive constants $C_{0}$ and $v$; here, $\|\cdot\|_{\mathrm{op}(X)}$ denotes the operator norm on $X$.

Under these assumptions, we define the extrapolation space $Z=X_{-1}$ with norm $\|z\|_{Z}=$ $\left\|A^{-1} z\right\|_{X}$ as the completion $\overline{\left(X,\|\cdot\|_{Z}\right)}$. Then $A_{-1}$, defined as the closure of $A$ in $X_{-1}$, is the unique continuous extension of the isometry $A: \mathcal{D}(A) \rightarrow X$ and yields an isometry $A_{-1}$ : $X=\mathcal{D}\left(A_{-1}\right) \subset X_{-1} \rightarrow X_{-1}$. The semigroup operators $e^{-t A}$ possess continuous extensions from $X$ to $X_{-1}$ defining an exponentially decaying analytic semigroup with infinitesimal generator $A_{-1}$; see Proposition 2.1. For simplicity, we will denote this semigroup by $e^{-t A}$ again. For details, we refer to [1, Chapter V, p. 262], [6, Chapter II.5]. If $X$ is reflexive, then $Z$ is isomorphic to $\left(\mathcal{D}\left(A^{\prime}\right)\right)^{\prime}$; see [1, Theorem V.1.4.6] or [6, Chapter II, Exercise 5.9(4)].

Hence, with an abuse of notation, we will write

$$
A: X \rightarrow Z=A X=\left(\mathcal{D}\left(A^{\prime}\right)\right)^{\prime}
$$

defining the isometry $\|A x\|_{Z}=\|x\|_{X}$ for $x \in X$.

For $1 \leq s \leq \infty$ and $\alpha \in \mathbb{R}$ such that $0<\alpha+\frac{1}{s}<1$, we define for $f \in Z$ the norm

$$
\|f\|_{\mathcal{X}_{s, \alpha}}:=\left\|A^{-1} f\right\|_{X}+ \begin{cases}\left(\int_{0}^{\infty} \tau^{\alpha s}\left\|e^{-\tau A} f\right\|_{X}^{s} \mathrm{~d} \tau\right)^{1 / s} & \text { when } s<\infty \\ \sup _{0<\tau<\infty} \tau^{\alpha}\left\|e^{-\tau A} f\right\|_{X} & \text { when } s=\infty\end{cases}
$$

and the space

$$
\mathcal{X}_{s, \alpha}=\left\{f \in Z:\|f\|_{s, \alpha}<\infty\right\} \text {. }
$$


We note that the term $\left\|A^{-1} f\right\|_{X}$ in (2.1) can be omitted. The idea well known from interpolation theory uses the identity $f=-\int_{0}^{\infty} \frac{\mathrm{d}}{\mathrm{d} \tau} e^{-\tau A} f \mathrm{~d} \tau=\int_{0}^{\infty} A e^{-\tau A} f \mathrm{~d} \tau$ in $Z$ which holds if $\left\|A e^{-\tau A} f\right\|_{Z}=\left\|e^{-\tau A} f\right\|_{X}$ is integrable on $(0, \infty)$. To control $\int_{0}^{\infty}\left\|e^{-\tau A} f\right\|_{X} \mathrm{~d} \tau$, we use Hölder's inequality and $\alpha s^{\prime}<1$ to see that

$$
\int_{0}^{1}\left\|e^{-\tau A} f\right\|_{X} \mathrm{~d} \tau \leq C\left(\int_{0}^{1} \tau^{\alpha s}\left\|e^{-\tau A} f\right\|_{X}^{S} \mathrm{~d} \tau\right)^{1 / s} ;
$$

moreover, by the exponential decay of $e^{-\tau A}$ an estimate similar to the above one holds also on $(1, \infty)$. Using the exponential decay of $e^{-\tau A}$ again and an argument by geometric series, we obtain that-for any $0<T<\infty$ - the norm in (2.1) can be replaced by the equivalent norm

$$
\|f\|_{\mathcal{X}_{s, \alpha}^{T}}= \begin{cases}\left(\int_{0}^{T} \tau^{\alpha s}\left\|e^{-\tau A} f\right\|_{X}^{s} \mathrm{~d} \tau\right)^{1 / s} & \text { when } s<\infty, \\ \sup _{0<\tau<T} \tau^{\alpha}\left\|e^{-\tau A} f\right\|_{X} & \text { when } s=\infty .\end{cases}
$$

To indicate which equivalent norm is used in $\mathcal{X}_{s, \alpha}$, we also use the notation $\mathcal{X}_{s, \alpha}^{T}$ instead of $\mathcal{X}_{s, \alpha}$ when $\mathcal{X}_{s, \alpha}$ is equipped with the norm $\|\cdot\|_{\mathcal{X}_{s, \alpha}^{T}}$.

From real interpolation theory applied to the spaces $Z$ and $X=\mathcal{D}\left(A_{-1}\right)$, for example, see [23, Proposition 6.2], we conclude that $\mathcal{X}_{s, \alpha}^{T}=\mathcal{X}_{s, \alpha}$ also coincides with the real interpolation space $(Z, X)_{1-\alpha-1 / s, s}$ endowed with the equivalent norm

$$
\|f\|_{(Z, X)_{1-\alpha-1 / s, s}}=\left(\int_{0}^{\infty}\left(\tau^{\alpha+1 / s}\left\|A e^{-\tau A} f\right\|_{Z}\right)^{s} \frac{\mathrm{d} \tau}{\tau}\right)^{1 / s},
$$

where the term $\left\|A^{-1} f\right\|_{X}$ is omitted. In the limit case when $s=\infty$, [23, Exercise 6.1.1 (1)] implies that $\mathcal{X}_{\infty, \alpha}^{T}=(Z, X)_{1-\alpha, \infty}$ for all $0<T \leq \infty$ with equivalent norms. Thus, for fixed $\theta=1-\alpha-\frac{1}{s} \in(0,1)$, i.e. $\alpha=\alpha(s)=1-\theta-\frac{1}{s} \in[0,1-\theta]$, we get the scale of interpolation spaces $(Z, X)_{\theta, s}$ for $\frac{1}{1-\theta}=$ : $s_{1} \leq s \leq \infty$ for all $0<T \leq \infty$ and with continuous embeddings

$$
X \subset(Z, X)_{\theta, s_{1}} \subset(Z, X)_{\theta, s} \subset(Z, X)_{\theta, \infty} \subset Z,
$$

or, since $(Z, X)_{\theta, s}=\mathcal{X}_{s, \alpha(s)}^{T}$, the scale $X \subset \mathcal{X}_{s_{1}, \alpha\left(s_{1}\right)}^{T} \subset \mathcal{X}_{s, \alpha(s)}^{T} \subset \mathcal{X}_{\infty, \alpha(\infty)}^{T} \subset Z$.

Proposition 2.1 (i) For $t>0$ and $f \in Z$, we have that $e^{-t A} f \in Z$ such that

$$
\left\|e^{-t A} f\right\|_{Z} \leq\left\|e^{-t A}\right\|_{\mathrm{op}(X)}\|f\|_{Z} .
$$

Moreover, $e^{-t A}$ extends to a bounded linear operator from $Z$ to $X$. To be more precise, there exists a constant $c>0$ independent of $t$ and $f \in Z$ such that

$$
\left\|e^{-t A} f\right\|_{X} \leq c t^{-1}\|f\|_{Z}, \quad t>0 .
$$

(ii) The space $X$ is continuously embedded into $\mathcal{X}_{s, \alpha}^{T}$ for all $\alpha \geq 0,1 \leq s \leq \infty$ and $0<T \leq \infty$.

Proof (i) By analyticity, we observe that for $f \in Z=X_{-1}$ and for $t>0$

$$
\begin{aligned}
\left\|e^{-t A} f\right\|_{Z} & =\left\|A_{-1}^{-1} e^{-t A} f\right\|_{X}=\left\|e^{-t A} A_{-1}^{-1} f\right\|_{X} \\
& \leq\left\|e^{-t A}\right\|_{\mathrm{op}(X)}\left\|A^{-1} f\right\|_{X} \leq\left\|e^{-t A}\right\|_{\mathrm{op}(X)}\|f\|_{Z} .
\end{aligned}
$$


If $f=A_{-1} x \in Z$ with $x \in X$, then

$$
\left\|e^{-t A} f\right\|_{X}=\left\|A_{-1} e^{-t A} x\right\|_{X} \leq c t^{-1}\|x\|_{X}=c t^{-1}\|f\|_{Z},
$$

with some constant $c$ independent of $f$ and $t$.

(ii) It is well known from real interpolation theory since $X \subset Z$; see also (2.4).

Besides the spaces $\mathcal{X}_{\infty, \alpha}$, we also need the closed subspace $\dot{\mathcal{X}}_{\infty, \alpha}$ defined by

$$
\dot{\mathcal{X}}_{\infty, \alpha}=\left\{f \in \mathcal{X}_{\infty, \alpha}: \sup _{0<\tau<\tau_{0}} \tau^{\alpha}\left\|e^{-\tau A} f\right\|_{X} \rightarrow 0 \text { as } \tau_{0} \rightarrow 0\right\} .
$$

By [23, Exercise 6.1.1 (1)], $\dot{\mathcal{X}}_{\infty, \alpha}$ coincides with the continuous interpolation space $(Z, X)_{1-\alpha, \infty}^{0}$ with equivalent norms. Thus, obviously $X \subset \dot{\mathcal{X}}_{\infty, \alpha} \subset \mathcal{X}_{\infty, \alpha} \subset Z$.

In view of (2.1), (2.2), we often suppress the $T$-dependence of $\mathcal{X}_{s, \alpha}^{T}$ and assume that $0<T<\infty$ is fixed. The notation $\mathcal{X}_{s, \alpha}^{T}$ is important in the construction of strong $L_{\alpha}^{s}\left(L^{q}\right)$ solution where $T$ yields a control of the interval of existence; see [8,9] and [11,12].

\section{Estimates of continuity}

In the following, we fix $0<T<\infty$ and simply write $\mathcal{X}_{s, \alpha}$ for $\mathcal{X}_{s, \alpha}^{T}$. The first continuity result considers the homogeneous part $e^{-t A} u_{0}$ in (1.7) and can be proved by general interpolation theory since by $(2.2),(2.4) \mathcal{X}_{s, \alpha}=A(X, \mathcal{D}(A))_{1-\alpha-\frac{1}{s}, s}=(Z, X)_{1-\alpha-\frac{1}{s}, s}$. However, we present a direct proof for completeness.

Proposition 3.1 Let $s \in[1, \infty]$ and $\alpha \geq 0$. Assume that $u_{0} \in \mathcal{X}_{s, \alpha}$.

(i) For $t \in(0, T]$, the estimate

$$
\left\|e^{-t A} u_{0}\right\|_{\mathcal{X}_{s, \alpha}^{T}} \leq C_{T}\left\|u_{0}\right\|_{\mathcal{X}_{s, \alpha}^{T}}
$$

holds with the constant $C_{T}=\sup _{t \in(0, T)}\left\|e^{-t A}\right\|_{\mathrm{op}(X)}$.

(ii) $e^{-t A} u_{0} \in C\left([0, \infty) ; \mathcal{X}_{s, \alpha}\right)$ if $u_{0} \in \mathcal{X}_{s, \alpha}$ and $s<\infty$.

(iii) $e^{-t A} u_{0} \in C\left([0, \infty) ; \dot{\mathcal{X}}_{\infty, \alpha}\right)$ if $u_{0} \in \dot{\mathcal{X}}_{\infty, \alpha}$.

(iv) For $u_{0} \in \mathcal{X}_{\infty, \alpha}$, continuity holds except at $t=0$, i.e. $e^{-t A} \in C\left((0, \infty) ; \dot{\mathcal{X}}_{\infty, \alpha}\right)$.

Moreover, $e^{-t A} u_{0} \stackrel{*}{\rightarrow} u_{0}$ as $t \rightarrow 0$ in $\mathcal{X}_{\infty, \alpha}$; for the latter result $X$ is assumed to be reflexive.

To prove Proposition 3.1, we use the strong continuity of the semigroup $e^{-t A}$ on $X$ and on $\mathcal{D}(A)$ near $t=0$.

Lemma 3.2 (i) $\left\|\left(e^{-t A}-\mathrm{I}\right) f\right\|_{X} \leq c_{\beta, T} t^{\beta}\left\|A^{\beta} f\right\|_{X}$ for each $\beta \in(0,1], t \in(0, T)$ and $f \in \mathcal{D}\left(A^{\beta}\right)$ with a constant $c_{\beta, T}>0$ independent of $f$ and $t>0$.

(ii) $\left\|\left(e^{-t A}-\mathrm{I}\right) e^{-\tau A} f\right\|_{X} \leq c_{\beta, T}\left(\frac{t}{\tau}\right)^{\beta}\|f\|_{X}$ for each $\beta \in(0,1], t \in(0, T)$ and $f \in X$ with $c_{\beta, T}$ independent of $t, \tau$ and $f$.

Proof of Lemma 3.2 (i) By the fundamental theorem of calculus,

$$
e^{-t A} f-f=-\int_{0}^{t} A e^{-\tau A} f \mathrm{~d} \tau=-\int_{0}^{t} A^{1-\beta} e^{-\tau A} A^{\beta} f \mathrm{~d} \tau .
$$


Since $\left\|A^{\lambda} e^{-t A}\right\|_{\mathrm{op}(X)} \leq c_{\lambda} \tau^{-\lambda}(\lambda>0)$ by analyticity, we observe that

$$
\left\|e^{-t A} f-f\right\|_{X} \leq c_{1-\beta} \int_{0}^{t} \frac{\mathrm{d} \tau}{\tau^{1-\beta}}\left\|A^{\beta} f\right\|_{X}=c_{\beta}^{\prime} t^{\beta}\left\|A^{\beta} f\right\|_{X} .
$$

(ii) This follows from (i) since $\left\|A^{\beta} e^{-\tau A}\right\|_{\mathrm{op}(X)} \leq c_{\beta} \tau^{-\beta}$.

Proof of Proposition 3.1 (i) This estimate is easy; for example, for $s<\infty$ we have

$$
\left\|e^{-t A} u_{0}\right\|_{\mathcal{X}_{s, \alpha}^{T}}^{s}=\int_{0}^{T} \tau^{\alpha s}\left\|e^{-(\tau+t) A} u_{0}\right\|_{X}^{s} \mathrm{~d} \tau \leq C_{T}^{s}\left\|u_{0}\right\|_{\mathcal{X}_{s, \alpha}^{T}}^{s}
$$

(ii) Let $t_{0}, t \geq 0$. Then

$$
\left\|e^{-t A} u_{0}-e^{-t_{0} A} u_{0}\right\|_{\mathcal{X}_{s, \alpha}^{T}}^{s}=\int_{0}^{T} \tau^{\alpha s}\left\|\left(e^{-t A}-e^{-t_{0} A}\right) e^{-\tau A} u_{0}\right\|_{X}^{s} \mathrm{~d} \tau
$$

converges to 0 as $t \rightarrow t_{0}$ by Lebesgue's theorem on dominated convergence since the integrand is uniformly estimated by an integrable function in $(0, T)$ and converges to 0 in the pointwise sense. This proves the continuity of $e^{-t A} u_{0}$ in $[0, \infty)$ with values in $\mathcal{X}_{s, \alpha}$.

(iii) Let $t, t_{0} \geq 0$. We take $\delta \in(0, T)$ and divide the supremum into two parts:

$$
\left\|e^{-t A} u_{0}-e^{-t_{0} A} u_{0}\right\|_{\mathcal{X}_{\infty, \alpha}^{T}} \leq\left(\sup _{\delta<\tau<T}+\sup _{0<\tau<\delta}\right) \tau^{\alpha}\left\|\left(e^{-t A}-e^{-t_{0} A}\right) e^{-\tau A} u_{0}\right\|_{X}=: J_{1}+J_{2} .
$$

Similarly to the case $s<\infty$, we observe that $J_{1} \rightarrow 0$ as $t \rightarrow t_{0}$. The second term is estimated as

$$
J_{2} \leq 2 C_{0} \sup _{0<\tau<\delta} \tau^{\alpha}\left\|e^{-\tau A} u_{0}\right\|_{X}
$$

If $u_{0} \in \dot{\mathcal{X}}_{\infty, \alpha}$, the right-hand side (which is independent of $t, t_{0}$ ) tends to zero as $\delta \rightarrow 0$. Thus, we conclude the continuity of $e^{-t A} u_{0}$ up to $t=0$ with values in $\dot{\mathcal{X}}_{\infty, \alpha}$.

(iv) If $u_{0} \in \mathcal{X}_{\infty, \alpha}$, the function $e^{-t A} u_{0}$ may not be continuous at $t=0$ with values in $\mathcal{X}_{\infty, \alpha}$. However, since $e^{-t A} u_{0} \in X$ by Proposition 2.1 for $t>0$ and $X \subset \dot{\mathcal{X}}_{s, \alpha}$, the assertion $e^{-t A} u_{0} \in C\left((0, \infty) ; \dot{\mathcal{X}}_{s, \alpha}\right)$ holds.

For the analysis at $t=0$, we apply the duality theorem of real interpolation, see [30, Theorem 1.11.2], and consider $\mathcal{X}_{\infty, \alpha}=(Z, X)_{1-\alpha, \infty}$ as the dual space of $\left(Z^{\prime}, X^{\prime}\right)_{1-\alpha, 1}=$ $\left(X^{\prime}, \mathcal{D}\left(A^{\prime}\right)\right)_{\alpha, 1}$ with the weighted norm $\int_{0}^{T} \tau^{-\alpha}\left\|A^{\prime} e^{-\tau A^{\prime}} \varphi\right\|_{X^{\prime}} \mathrm{d} \tau$ for $\varphi \in\left(X^{\prime}, \mathcal{D}\left(A^{\prime}\right)\right)_{\alpha, 1}, c f$. (2.3). Given $\varphi$ we get that

$$
\begin{aligned}
\left|\left\langle e^{-t A} u_{0}-u_{0}, \varphi\right\rangle\right| & =\left|\left\langle u_{0}, e^{-t A^{\prime}} \varphi-\varphi\right\rangle\right| \\
& \leq\left\|u_{0}\right\|_{\mathcal{X}_{\infty, \alpha}}\left\|e^{-t A^{\prime}} \varphi-\varphi\right\|_{\left(X^{\prime}, \mathcal{D}\left(A^{\prime}\right)\right)_{\alpha, 1}} .
\end{aligned}
$$

To show that the latter term converges to 0 as $t \rightarrow 0$ we note that part (ii) of this proposition holds also for negative $\alpha$ as is easily seen.

To estimate nonlinear terms as on the right-hand side of (1.7), we consider for $\mu>0$ the integral operator

$$
(N f)(t)=\int_{0}^{t} A^{\mu} e^{-(t-\tau) A} f(\tau) \mathrm{d} \tau
$$


for $f \in L_{\alpha_{1}}^{s_{1}}(0, T ; Y)$. Here $Y$ is another Banach space containing $X$ and $e^{-t A}$ can be extended to $Y$ having a regularizing estimate

$$
\left\|e^{-t A} a\right\|_{X} \leq c_{T} t^{-\eta}\|a\|_{Y}, \quad a \in Y, \quad t \in(0, T)
$$

for some $\eta>0$ with $c_{T}$ independent of $a$.

We recall the weighted Hardy-Littlewood-Sobolev inequality [28,29]. The limit cases $s_{1}, s_{2} \in\{1, \infty\}$ are considered in [29, Theorem 5].

Lemma 3.3 Assume that $\lambda \in(0,1)$ satisfies the scale balance of exponents $1 / s_{1}+\alpha_{1}+\lambda=$ $1 / s_{2}+\alpha_{2}+1$ under the restrictions of exponents $1 \leq s_{1} \leq s_{2} \leq \infty, \alpha_{2} \leq \alpha_{1}$ and $0<\alpha_{1}+1 / s_{1}<1,0<\alpha_{2}+1 / s_{2}<1$. Then the integral operator

$$
\left(I_{\lambda} f\right)(t)=\int_{\mathbb{R}}|t-\tau|^{-\lambda} f(\tau) \mathrm{d} \tau
$$

is bounded from $L_{\alpha_{1}}^{s_{1}}(\mathbb{R})$ to $L_{\alpha_{2}}^{s_{2}}(\mathbb{R})$. If $\alpha_{2}=\alpha_{1}$, then the stricter condition $1<s_{1}, s_{2}<\infty$ is needed.

By $\left\|A^{\mu} e^{-t A}\right\|_{\mathrm{op}(X)} \leq C t^{-\mu}$ and (3.2),

$$
\|(N f)(t)\|_{X} \leq C \int_{0}^{t}(t-\tau)^{-\mu-\eta}\|f(\tau)\|_{Y} \mathrm{~d} \tau,
$$

so that Lemma 3.3 yields the following:

Proposition 3.4 Assume that $\lambda=\mu+\eta \in(0,1)$ for positive $\mu, \eta$ as in (3.1), (3.2). Then $N$ defined by (3.1) is a bounded operator from $L_{\alpha_{1}}^{s_{1}}(0, T ; Y)$ to $L_{\alpha_{2}}^{s_{2}}(0, T ; X)$. Here the exponents $s_{j}, \alpha_{j}$ satisfy the assumptions in Lemma 3.3, and the operator norm of $N$ is independent of $T$.

We claim that $N f(\cdot)$ belongs to $C\left([0, T] ; \mathcal{X}_{s_{2}, \alpha_{2}}\right)$ and start with the case $s_{2}<\infty$.

Theorem 3.5 Assume that $\lambda=\mu+\eta \in(0,1)$ for positive $\mu, \eta$ as in (3.1), (3.2) satisfies the scale balance $1 / s_{1}+\alpha_{1}+\lambda=1 / s_{2}+\alpha_{2}+1$ for exponents $1<s_{1} \leq s_{2}<\infty, \alpha_{2} \leq \alpha_{1}$ where $0<\alpha_{1}+1 / s_{1}<1,0<\alpha_{2}+1 / s_{2}<1$. If $f \in L_{\alpha_{1}}^{s_{1}}(0, T ; Y)$, then

$$
\|N f(t)\|_{\mathcal{X}_{s_{2}, \alpha_{2}}^{T}} \leq C\|f\|_{L_{\alpha_{1}}^{s_{1}}(0, t ; Y)}, \quad t \in[0, T] .
$$

Moreover,

$$
N f \in C\left([0, T] ; \mathcal{X}_{s_{2}, \alpha_{2}}\right) .
$$

Proof By definition, we get from (3.3) that

$$
\begin{aligned}
\|N f(t)\|_{\mathcal{X}_{s_{2}, \alpha_{2}}^{T}} & =\left(\int_{0}^{T} \tau^{\alpha_{2} s_{2}}\left\|e^{-\tau A}(N f)(t)\right\|_{X}^{s_{2}} \mathrm{~d} \tau\right)^{1 / s_{2}} \\
& \leq C\left(\left\|\int_{0}^{t}(t+\tau-\rho)^{-\mu-\eta}\right\| f(\rho)\left\|_{Y} \mathrm{~d} \rho\right\|_{L_{\alpha_{2}}^{s_{2}}(0, T)}^{s_{2}}\right)^{1 / s_{2}} \\
& =C\left(\int_{0}^{T}\left(\tau^{\alpha_{2}} \int_{\mathbb{R}}|t+\tau-\rho|^{-\lambda}\|(f \chi)(\rho)\|_{Y} \mathrm{~d} \rho\right)^{s_{2}} \mathrm{~d} \tau\right)^{1 / s_{2}}
\end{aligned}
$$


with $\chi=\chi_{(0, t)}$, the characteristic function of the interval $(0, t)$. Using the change of variables $\tau^{\prime}=\tau+t$ and that $0 \leq \tau^{\prime}-t \leq \tau^{\prime}$ Lemma 3.3 implies that

$$
\begin{aligned}
\|N f(t)\|_{\mathcal{X}_{s_{2}, \alpha_{2}}^{T}} & \leq C\left(\int_{t}^{t+T}\left(\left(\tau^{\prime}-t\right)^{\alpha_{2}} \int_{\mathbb{R}}\left|\tau^{\prime}-\rho\right|^{-\lambda}\|(f \chi)(\rho)\|_{Y} \mathrm{~d} \rho\right)^{s_{2}} \mathrm{~d} \tau^{\prime}\right)^{1 / s_{2}} \\
& \leq C\left\|I_{\lambda}\left(\|f \chi\|_{Y}\right)\right\|_{L_{\alpha_{2}}^{s_{2}}(t, t+T)} \\
& \leq C\|\| f \chi\left\|_{Y}\right\|_{L_{\alpha_{1}}^{s_{1}}}=C\|f\|_{L_{\alpha_{1}}^{s_{1}}(0, t ; Y)} .
\end{aligned}
$$

The proof of continuity is based on the previous estimates. By definition for $t_{1} \geq t_{2} \geq 0$, we observe that

$$
\begin{aligned}
& (N f)\left(t_{1}\right)-(N f)\left(t_{2}\right) \\
& =\int_{t_{2}}^{t_{1}} A^{\mu} e^{-\left(t_{1}-\rho\right) A} f(\rho) \mathrm{d} \rho+\int_{0}^{t_{2}}\left(A^{\mu} e^{-\left(t_{1}-\rho\right) A}-A^{\mu} e^{-\left(t_{2}-\rho\right) A}\right) f(\rho) \mathrm{d} \rho \\
& =: \mathcal{I}_{1}+\mathcal{I}_{2} .
\end{aligned}
$$

The first term is easy to estimate. Replacing $f$ by $f \chi_{\left(t_{2}, t_{1}\right)}$ and rewriting $\mathcal{I}_{1}$ as an integral for $f \chi_{\left(t_{2}, t_{1}\right)}(\rho)$ with $\rho \in\left(0, t_{1}\right),(3.4)$ proves that

$$
\begin{aligned}
\left\|\mathcal{I}_{1}\right\|_{\mathcal{X}_{s_{2}, \alpha_{2}}^{T}} & \leq C\left\|\int_{0}^{t_{1}}\left(t_{1}+\tau-\rho\right)^{-\mu-\eta}\right\| f(\rho) \chi_{\left(t_{2}, t_{1}\right)}(\rho)\left\|_{Y} \mathrm{~d} \rho\right\|_{L_{\alpha_{2}}^{s_{2}(0, T)}} \\
& \leq C\|f\|_{L_{\alpha_{1}}^{s_{1}\left(t_{2}, t_{1} ; Y\right)}} \rightarrow 0
\end{aligned}
$$

as $t_{1}-t_{2} \rightarrow 0$. The integral $\mathcal{I}_{2}$ is divided into two parts:

$$
\left\|\mathcal{I}_{2}\right\|_{\mathcal{X}_{s_{2}, \alpha_{2}}^{T}}^{s_{2}}=\int_{0}^{T} \tau^{\alpha_{2} s_{2}}\left\|e^{-\tau A} \mathcal{I}_{2}\right\|_{X}^{s_{2}} \mathrm{~d} \tau=\left(\int_{0}^{\delta}+\int_{\delta}^{T}\right) \tau^{\alpha_{2} s_{2}}\left\|e^{-\tau A} \mathcal{I}_{2}\right\|_{X}^{s_{2}} \mathrm{~d} \tau .
$$

The first part is estimated as follows: for $t_{1,2}=t_{1}$ and $t_{1,2}=t_{2}$,

$$
\begin{aligned}
& C \int_{0}^{\delta} \tau^{\alpha_{2} s_{2}}\left\|\int_{0}^{t_{2}} A^{\mu} e^{-\left(t_{1,2}+\tau-\rho\right) A} f(\rho) \mathrm{d} \rho\right\|_{X}^{s_{2}} \mathrm{~d} \tau \\
& \quad \leq C \int_{0}^{\delta} \tau^{\alpha_{2} s_{2}}\left(\int_{0}^{t_{2}}\left(t_{2}+\tau-\rho\right)^{-\lambda}\|f(\rho)\|_{Y} \mathrm{~d} \rho\right)^{s_{2}} \mathrm{~d} \tau
\end{aligned}
$$

for $t_{1}$, we used that $\left(t_{1}+\tau-\rho\right)^{-\lambda} \leq\left(t_{2}+\tau-\rho\right)^{-\lambda}$ since $t_{1} \geq t_{2}$. Replacing $\delta$ by $T$, we conclude — as for the estimate of $\left\|\mathcal{I}_{1}\right\|_{\mathcal{X}_{s_{2}, \alpha_{2}}^{T}}$-from Lemma 3.3 that the right-hand double integral is bounded by $C\|f\|_{L_{\alpha_{1}}^{s_{1}\left(0, t_{2} ; Y\right)}}^{s_{2}}$. Hence, as a function of $\delta$, the right-hand side converges to 0 as $\delta \rightarrow 0$, uniformly in $0 \leq t_{2} \leq t_{1} \leq T$.

To estimate the integral over $(\delta, T)$ in $\left\|\mathcal{I}_{2}\right\|_{\mathcal{X}_{s_{2}, \alpha_{2}}^{T}}^{s_{2}}$, we observe that

$$
\int_{\delta}^{T} \tau^{\alpha_{2} s_{2}}\left\|e^{-\tau A} \mathcal{I}_{2}\right\|_{X}^{s_{2}} \mathrm{~d} \tau=\int_{\delta}^{T} \tau^{\alpha_{2} s_{2}} \varphi\left(\tau, t_{1}, t_{2}\right) \mathrm{d} \tau,
$$

where by Lemma 3.2 (ii) for any $v_{1} \in(0,1)$

$$
\begin{aligned}
\varphi\left(\tau, t_{1}, t_{2}\right) & =\left\|\int_{0}^{t_{2}}\left(e^{-\left(t_{1}-t_{2}\right) A}-\mathrm{I}\right) e^{-\tau A} A^{\mu} e^{-\left(t_{2}-\rho\right) A} f(\rho) \mathrm{d} \rho\right\|_{X}^{s_{2}} \\
& \leq C\left|\frac{t_{2}-t_{1}}{\tau}\right|^{\nu_{1} s_{2}}\left(\int_{0}^{t_{2}}\left\|e^{-\tau A / 2} A^{\mu} e^{-\left(t_{2}-\rho\right) A} f(\rho)\right\|_{X} \mathrm{~d} \rho\right)^{s_{2}} .
\end{aligned}
$$


Thus,

$$
\begin{aligned}
& \int_{\delta}^{T} \tau^{\alpha_{2} s_{2}} \varphi\left(\tau, t_{1}, t_{2}\right) \mathrm{d} \tau \\
& \quad \leq C\left|\frac{t_{2}-t_{1}}{\delta}\right|^{\nu_{1} s_{2}} \int_{0}^{T}\left(\int_{0}^{t_{2}}\left(t_{2}+\frac{\tau}{2}-\rho\right)^{-\lambda}\|f(\rho)\|_{Y} \mathrm{~d} \rho\right)^{s_{2}} \mathrm{~d} \tau \\
& \quad \leq C\left|\frac{t_{2}-t_{1}}{\delta}\right|^{\nu_{1} s_{2}}\|f\|_{L_{\alpha_{1}}^{s_{2}}\left(0, t_{2} ; Y\right)}^{s_{2}}
\end{aligned}
$$

converges to 0 as $t_{2}-t_{1} \rightarrow 0$ for fixed $\delta>0$.

Now the proof of continuity in the case of finite $s_{2}$ is complete.

Next we handle the case $\mathcal{X}_{\infty, \alpha}$.

Theorem 3.6 Assume that $\lambda=\mu+\eta \in(0,1)$ for positive $\mu, \eta$ as in (3.1), (3.2), and that $0<\alpha_{2}=\lambda+\alpha_{1}-1,0<\alpha_{1}<1$. Let $f \in L_{\alpha_{1}}^{\infty}(0, T ; Y)$ satisfy the condition

$$
\|f\|_{L_{\alpha_{1}}^{\infty}(t)}:=\|f\|_{L_{\alpha_{1}}^{\infty}(0, t ; Y)} \rightarrow 0 \text { as } t \rightarrow 0 .
$$

(i) For $t \in(0, T)$,

$$
\|N f(t)\|_{\mathcal{X}_{\infty, \alpha_{2}}^{T}} \leq C_{T}\|f\|_{L_{\alpha_{1}}^{\infty}(t)}
$$

Particularly, $N f(t) \rightarrow 0$ as $t \rightarrow 0$ in $\mathcal{X}_{\infty, \alpha_{2}}$ and $N f(t) \in \stackrel{\circ}{\mathcal{X}}_{\infty, \alpha_{2}}$.

(ii) $N f \in C\left([0, T], \stackrel{\circ}{\mathcal{X}}_{\infty, \alpha_{2}}\right)$.

Proof (i) We first observe, by (3.2) and the analyticity of $e^{-t A}$, that for $0 \leq \tau<T$

$$
\tau^{\alpha_{2}}\left\|e^{-\tau A} N f(t)\right\|_{X} \leq C \tau^{\alpha_{2}} \int_{0}^{t}(t+\tau-\rho)^{-\lambda} \rho^{-\alpha_{1}} \mathrm{~d} \rho\|f\|_{L_{\alpha_{1}}^{\infty}(t)} .
$$

Thus, for $t \leq \tau<T$,

$$
\begin{aligned}
\sup _{t \leq \tau<T} \tau^{\alpha_{2}}\left\|e^{-\tau A} N f(t)\right\|_{X} & \leq C \sup _{t \leq \tau<T} \tau^{\alpha_{2}} \int_{0}^{\tau}(\tau-\rho)^{-\lambda} \rho^{-\alpha_{1}} \mathrm{~d} \rho\|f\|_{L_{\alpha_{1}}^{\infty}(t)} \\
& \leq C B\|f\|_{L_{\alpha_{1}}^{\infty}(t)}
\end{aligned}
$$

by the scale balance, where $B=B\left(1-\lambda, 1-\alpha_{1}\right)$ is the beta function. For $\tau \leq t$, we have

$$
\begin{aligned}
\sup _{0<\tau<t} \tau^{\alpha_{2}}\left\|e^{-\tau A} N f(t)\right\|_{X} & \leq C \sup _{0<\tau<t} \tau^{\alpha_{2}} \int_{0}^{t}(t-\rho)^{-\lambda} \rho^{-\alpha_{1}} \mathrm{~d} \rho\|f\|_{L_{\alpha_{1}}^{\infty}(t)} \\
& =C B \sup _{0<\tau<t} \tau^{\alpha_{2}} t^{-\alpha_{2}}\|f\|_{L_{\alpha_{1}}^{\infty}(t)} \\
& =C B\|f\|_{L_{\alpha_{1}}^{\infty}(t) .}
\end{aligned}
$$

Hence, under assumption (3.6),

$$
\|N f(t)\|_{\mathcal{X}_{\infty, \alpha_{2}}^{T}} \leq C B\|f\|_{L_{\alpha_{1}}^{\infty}(t)} \rightarrow 0 \text { as } t \rightarrow 0 .
$$

For fixed $t>0$, a modification of (3.8) also yields for $0<\tau<\tau_{0}<t$ the estimate

$$
\sup _{0<\tau<\tau_{0}} \tau^{\alpha_{2}}\left\|e^{-\tau A} N f(t)\right\|_{X} \leq C(t) \sup _{0<\tau<\tau_{0}} \tau^{\alpha_{2}} \cdot\|f\|_{L_{\alpha_{1}}^{\infty}(t)}
$$

i.e. $N f(t) \in \stackrel{\circ}{\mathcal{X}}_{\infty, \alpha_{2}}$. 
(ii) It remains to prove the continuity of $N f(t)$ in $\mathcal{X}_{\infty, \alpha_{2}}$ for $t \geq \delta>0$ for arbitrary $\delta>0$. By definition for $t_{1} \geq t_{2} \geq \delta>0$, we observe that

$$
\begin{aligned}
(N f)\left(t_{1}\right) & -(N f)\left(t_{2}\right) \\
& =\int_{t_{2}}^{t_{1}} A^{\mu} e^{-\left(t_{1}-\rho\right) A} f(\rho) \mathrm{d} \rho+\int_{0}^{t_{2}}\left(e^{-\left(t_{1}-t_{2}\right) A}-\mathrm{I}\right) A^{\mu} e^{-\left(t_{2}-\rho\right) A} f(\rho) \mathrm{d} \rho \\
& =: \mathcal{I}_{1}+\mathcal{I}_{2} .
\end{aligned}
$$

The term $\mathcal{I}_{1}$ is easy to treat. Due to the boundedness of the operator family $e^{-\tau A}, 0 \leq$ $\tau \leq T$, on $X$ it suffices to consider $\left\|\mathcal{I}_{1}\right\|_{X}$ directly. If $0<\tau<T$,

$$
\begin{aligned}
\left\|\mathcal{I}_{1}\right\|_{X} & \leq c \int_{t_{2}}^{t_{1}}\left(t_{1}-\rho\right)^{-\lambda} \rho^{-\alpha_{1}} \mathrm{~d} \rho\|f\|_{L_{\alpha_{1}}^{\infty}(T)} \\
& \leq c_{\delta} \int_{t_{2}}^{t_{1}}\left(t_{1}-\rho\right)^{-\lambda} \mathrm{d} \rho\|f\|_{L_{\alpha_{1}}^{\infty}(T)} \\
& \leq C_{\delta}\left(t_{1}-t_{2}\right)^{1-\lambda}\|f\|_{L_{\alpha_{1}}^{\infty}(T)} .
\end{aligned}
$$

Thus,

$$
\limsup _{\substack{t_{1}-t_{2} \rightarrow 0 \\ t_{1}, t_{2} \geq \delta}} \sup _{\substack{0<\tau<T \\ n^{2}}} \tau^{\alpha_{2}}\left\|e^{-\tau A} \mathcal{I}_{1}\right\|_{X}=0 .
$$

For the estimate of $\mathcal{I}_{2}$, we consider $\left\|\mathcal{I}_{2}\right\|_{X}$ directly. By Lemma 3.2 (ii) and with $0<\beta<$ $1-\lambda$,

$$
\begin{aligned}
\left\|\mathcal{I}_{2}\right\|_{X} & \leq c \int_{0}^{t_{2}}\left(\frac{t_{1}-t_{2}}{t_{2}-\rho}\right)^{\beta}\left(t_{2}-\rho\right)^{-\lambda}\|f(\rho)\|_{Y} \mathrm{~d} \rho \\
& \leq c\left(t_{1}-t_{2}\right)^{\beta} \int_{0}^{t_{2}}\left(t_{2}-\rho\right)^{-\lambda-\beta} \rho^{-\alpha_{1}} \mathrm{~d} \rho\|f\|_{L_{\alpha_{1}}^{\infty}(T)} \\
& \leq c_{\delta}\left(t_{1}-t_{2}\right)^{\beta}\|f\|_{L_{\alpha_{1}}^{\infty}(T)}
\end{aligned}
$$

since $t_{2} \geq \delta>0$. We thus conclude that

$$
\limsup _{\substack{t_{1}-t_{2} \rightarrow 0 \\ t_{1}, t_{2} \geq \delta}} \sup _{\substack{0<\tau<T \\ 0 .}}\left\|\tau^{\alpha_{2}} e^{-\tau A} \mathcal{I}_{2}\right\|_{X}=0 .
$$

Now the assertion $N f \in C\left((0, T] ; \mathcal{X}_{\infty, \alpha_{2}}\right)$ is proved.

\section{Proof of main theorems}

We shall prove Theorems 1.1 and 1.2 based on the abstract results given in the previous section.

Proof of Theorem 1.1 We first note that if $X=L_{\sigma}^{q}(\Omega), A$ is taken as the Stokes operator and if $2 / s+3 / q=1-2 \alpha$, the Besov space $\mathbb{B}_{q, s}^{-1+3 / q}$ is identical with the weighted space $\mathcal{X}_{s, \alpha}^{T}=\mathcal{X}_{s, \alpha}$ (for all $0<T \leq \infty$ ) with equivalent norms; see Sect. 2 and Theorem 5.1. For $u_{0} \in \mathbb{B}_{q, s}^{-1+3 / q}$, the $L_{\alpha}^{s}\left(L^{q}\right)$-strong solution $u$ satisfies the integral equation 


$$
\begin{aligned}
u(t) & =e^{-t A} u_{0}-\int_{0}^{t} e^{-(t-\rho) A} \mathbb{P} \nabla \cdot((u \otimes u)(\rho)-F(\rho)) \mathrm{d} \rho \\
& =e^{-t A} u_{0}-\int_{0}^{t} A^{1 / 2} e^{-(t-\rho) A} A^{-1 / 2} \mathbb{P} \nabla \cdot((u \otimes u)(\rho)-F(\rho)) \mathrm{d} \rho,
\end{aligned}
$$

where $A^{-1 / 2} \mathbb{P} \nabla$ is bounded in any $L^{r}(\Omega)$-space, $1<r<\infty$ (see Giga and Miyakawa [15] and Sohr [26]). We observe from the assumptions $u \in L_{\sigma}^{s}\left(L^{q}\right)$ and $F \in L_{2 \alpha}^{s / 2}\left(L^{q / 2}\right)$ that

$$
f:=A^{-1 / 2} \mathbb{P} \nabla \cdot((u \otimes u)-F) \in L_{2 \alpha}^{s / 2}\left(0, T ; L_{\sigma}^{q / 2}(\Omega)\right) .
$$

We take $Y=L_{\sigma}^{q / 2}(\Omega)$ and $X=L_{\sigma}^{q}(\Omega)$ and rewrite (4.1) as

$$
u(t)=e^{-t A} u_{0}+N f(t)
$$

with $\mu=1 / 2$. By Proposition 3.1, $e^{-t A} u_{0} \in C\left([0, \infty), X_{s, \alpha}\right)$. Since, see [15],

$$
\left\|e^{-t A} v\right\|_{X} \leq C_{T} t^{-\eta}\|v\|_{Y}
$$

with $\eta=3 / 2 q$, the operator $N$ satisfies assumptions (3.1), (3.3) with $\mu=1 / 2, \eta=3 / 2 q$. Thus, Theorem 3.5 implies that $N f \in C\left([0, \infty), \mathcal{X}_{s, \alpha}\right)$.

Proof of Theorem 1.2 Obviously, the condition $u_{0} \in \mathbb{B}_{q, \infty}^{-1+3 / q}$ is equivalent to say that $u_{0} \in$ $\stackrel{\circ}{\mathcal{X}}_{\infty, \alpha}$ with $3 / q=1-2 \alpha$. We recall the construction of the solution of (4.1) by the iteration

$$
\begin{aligned}
u_{1}(t) & =e^{-t A} u_{0}, \\
u_{m+1}(t) & =e^{-t A} u_{0}-\int_{0}^{t} A^{1 / 2} e^{-(t-\rho) A} A^{-1 / 2} \mathbb{P} \nabla \cdot\left(u_{m} \otimes u_{m}-F\right)(\rho) \mathrm{d} \rho \quad(m \geq 1) .
\end{aligned}
$$

Since $u_{0} \in \dot{\mathcal{X}}_{\infty, \alpha}$, by Proposition 3.1 (iii) $u_{1}(t)=e^{-t A} u_{0} \in C\left([0, T] ; \dot{\mathcal{X}}_{\infty, \alpha}\right)$ and even $\left\|u_{1}\right\|_{L_{\alpha}^{\infty}(0, t ; X)} \rightarrow 0$ as $t \rightarrow 0$. By the assumption on $F$ in Theorem 1.2, we conclude from Proposition 3.4 inductively that $\left\|u_{m+1}\right\|_{L_{\alpha}^{\infty}(0, t ; X)} \rightarrow 0$ as $t \rightarrow 0$ for every $m \in \mathbb{N}$. Hence, also the limit solution $u$ which is the limit of $\left(u_{m}\right)$ in $L_{\alpha}^{\infty}(0, T ; X)$ has the same property at $t=0$.

We now consider (4.1) and apply Proposition 3.1 and Theorem 3.6, under assumption (3.6) satisfied by $u, F$, to get the desired continuity.

Proof of Theorem 1.3 Let $u, v$ be $L_{\alpha}^{s}\left(L^{q}\right)$-strong solutions of (1.1) with data $f=\operatorname{div} F, u_{0}$ and $g=\operatorname{div} G, v_{0}$. Being mild solutions of (4.1), the difference $w=u-v$ solves the integral equation

$$
\begin{aligned}
w(t) & =e^{-t A} w_{0}-\int_{0}^{t} A^{1 / 2} e^{-(t-\rho) A} A^{-1 / 2} \mathbb{P} \nabla \cdot(w \otimes u+v \otimes w-(F-G))(\rho) \mathrm{d} \rho \\
& =e^{-t A} w_{0}-(N g)(t),
\end{aligned}
$$

where the linear operator $N$ is defined by (3.1) with $\mu=\frac{1}{2}, X=L^{q}(\Omega), Y=L^{q / 2}(\Omega)$, $\eta=\frac{3}{2 q}$ in (3.2), and

$$
g=A^{-1 / 2} \mathbb{P} \nabla \cdot(w \otimes u+v \otimes w-(F-G))
$$

satisfies the elementary estimate

$$
\|g\|_{L_{2 \alpha}^{s / 2}\left(L^{q / 2}\right)} \leq c\left(\|w\|_{L_{\alpha}^{s}\left(L^{q}\right)}\left(\|u\|_{L_{\alpha}^{s}\left(L^{q}\right)}+\|v\|_{L_{\alpha}^{s}\left(L^{q}\right)}\right)+\|F-G\|_{L_{2 \alpha}^{s / 2}\left(L^{q / 2}\right)}\right) .
$$


Now Lemma 3.3 with exponents

$$
s_{1}=\frac{s}{2}, \alpha_{1}=2 \alpha, s_{2}=s, \alpha_{2}=\alpha \quad \text { and } \lambda=\frac{1}{2}+\frac{3}{2 q}
$$

implies that

$$
\|N g\|_{L_{\alpha_{2}}^{s_{2}\left(L^{q}\right)}} \leq C\|g\|_{L_{\alpha_{1}}^{s_{1}\left(L^{q / 2}\right)}} .
$$

Since $\left\|e^{-t A} w_{0}\right\|_{L_{\alpha}^{s}\left(L^{q}\right)} \leq\left\|w_{0}\right\|_{\mathcal{X}_{s, \alpha}^{T}}$, Proposition 3.4 and (4.3) lead to the estimate

$$
\|w\|_{L_{\alpha}^{s}\left(L^{q}\right)} \leq\left\|w_{0}\right\|_{\mathcal{X}_{s, \alpha}^{T}}+C\left(\|w\|_{L_{\alpha}^{s}\left(L^{q}\right)}\left(\|u\|_{L_{\alpha}^{s}\left(L^{q}\right)}+\|v\|_{L_{\alpha}^{s}\left(L^{q}\right)}\right)+\|F-G\|_{L_{2 \alpha}^{s / 2}\left(L^{q / 2}\right)}\right)
$$

where the $L_{\alpha}^{s}\left(L^{q}\right)$-norm is considered on a time interval $(0, T)$. Choosing $T_{0} \leq T$ such that, as in the assumption of Theorem 1.3, $\|u, v\|_{L_{\alpha}^{s}\left(0, T_{0} ; L^{q}\right)} \leq \varepsilon_{*}$ with $2 C \varepsilon_{*} \leq \frac{1}{2}$ the term involving $w$ on the right-hand side of (4.6) can be absorbed. This proves the estimate (1.4), but still with the left-hand side replaced by $\|u-v\|_{L_{\alpha}^{s}\left(L^{q}\right)}$.

Next we apply (3.4) from Theorem 3.5 to (4.2) to get that

$$
\begin{aligned}
\|w(t)\|_{\mathcal{X}_{s, \alpha}^{T} \leq} & \left\|u_{0}-v_{0}\right\|_{\mathcal{X}_{s, \alpha}^{T}}+\|N g(t)\|_{\mathcal{X}_{s, \alpha}^{T}} \\
\leq & \left\|u_{0}-v_{0}\right\|_{\mathcal{X}_{s, \alpha}^{T}}+C\|g\|_{L_{2 \alpha}^{s / 2}\left(0, T ; L^{q / 2}\right)} \\
\leq & \left\|u_{0}-v_{0}\right\|_{\mathcal{X}_{s, \alpha}^{T}}+C\|F-G\|_{L_{2 \alpha}^{s / 2}\left(0, T ; L^{q / 2}\right)} \\
& +C\|w\|_{L_{\alpha}^{s}\left(0, T ; L^{q}\right)}\left(\|u\|_{L_{\alpha}^{s}\left(0, T ; L^{q}\right)}+\|v\|_{L_{\alpha}^{s}\left(0, T ; L^{q}\right)}\right) .
\end{aligned}
$$

Under the smallness assumption on $\|u, v\|_{L_{\alpha}^{s}\left(0, T_{0} ; L^{q}\right)}$ for a suitable $0<T_{0} \leq T$, we can insert (4.6), i.e. (1.4) with the left-hand side $\|u-v\|_{L_{\alpha}^{s}\left(0, T_{0} ; L^{q}\right)}$ instead of $\|u-v\|_{\mathcal{X}_{s, \alpha}^{T}}$, into (4.7) and conclude the proof of Theorem 1.3.

Proof of Theorem 1.4 The proof is similar to the proof of Theorem 1.3. Here $s_{1}^{\prime}=s_{2}^{\prime}=1$ and (3.7) from Theorem 3.6 is applied to (4.6).

Remark 4.1 (i) Our continuity results (Theorems 1.1 and 1.2) have an overlap with results in [16, Remark 4.19, Theorem 4.20]. Their external force $f$ is allowed to be of the form $f=f_{0}+\operatorname{div} F$, where both $f$ and $F$ are $t$-dependent but with values in $L^{2}(\Omega)$. If there are no external forces, our results are contained in their results. However, in [16] a heavy, technical machinery based on interpolation and extrapolation spaces is applied to abstract parabolic equations with quadratic nonlinearity. Our approach is much more direct and simple and uses only basic terminology from interpolation theory including — in one step - the duality theorem of real interpolation.

(ii) The results of Amann [2] cannot be compared with ours. In [2, Sect. 5], he considers more regular solutions with initial value in $\stackrel{\circ}{B}_{q, \infty}^{-1+3 / q}$ but with forces in weighted $C^{0}$ spaces so that solutions are classically regular for $t>0$; see [2, Theorem 6.1]. Although a weaker force is discussed in [2, Remark 7.3], his space is not of Besov type but of Bessel potential type in the analysis of the Navier-Stokes system on a domain.

\section{Appendix: Besov spaces}

For $1<q<\infty, 1 \leq r \leq \infty$ and $t \in \mathbb{R}$, let $B_{q, r}^{t}\left(\mathbb{R}^{3}\right)$ denote the usual Besov spaces, see [30, 2.3.1], and define for the bounded domain $\Omega \subset \mathbb{R}^{3}$ the space $B_{q, r}^{t}(\Omega)$ by restriction of 
elements in $B_{q, r}^{t}\left(\mathbb{R}^{3}\right)$ in the sense of distributions to $\Omega$; the norm of $u \in B_{q, r}^{t}(\Omega)$ is defined by $\|u\|_{B_{q, r}^{t}(\Omega)}=\inf \left\{\|v\|_{B_{q, r}^{t}\left(\mathbb{R}^{3}\right)}: v \in B_{q, r}^{t}\left(\mathbb{R}^{3}\right), v_{\left.\right|_{\Omega}}=u\right\}$. Concerning Besov spaces on $\Omega$ with vanishing trace-if possible - the definition is modified as follows: Considering only vector fields rather than scalar-valued functions and the range $t \in[-2,2]$, we follow Amann $[2,3]$ and define

$$
\mathbf{B}_{q, r}^{t}(\Omega)= \begin{cases}\left\{u \in B_{q, r}^{t}(\Omega)^{3} ; u_{\mid \partial \Omega}=0\right\}, & 1 / q<t \leq 2, \\ \left\{u \in B_{q, r}^{1 / q}\left(\mathbb{R}^{3}\right)^{3} ; \operatorname{supp}(u) \subset \bar{\Omega}\right\}, & 1 / q=t, \\ B_{q, r}^{t}(\Omega)^{3}, & 0 \leq t<1 / q, \\ \left(\mathbf{B}_{q^{\prime}, r^{\prime}}^{-t}(\Omega)\right)^{\prime}(1<r \leq \infty), & -2 \leq t<0 .\end{cases}
$$

For spaces of solenoidal vector fields on $\Omega$, let

$$
\mathbb{B}_{q, r}^{t}(\Omega)= \begin{cases}\mathbf{B}_{q, r}^{t}(\Omega) \cap L_{\sigma}^{q}(\Omega), & 0<t \leq 2, \\ \operatorname{cl}\left(C_{c, \sigma}^{\infty}(\Omega)\right) \text { in } \mathbf{B}_{q, r}^{0}(\Omega), & t=0, \\ \left(\mathbb{B}_{q^{\prime}, r^{\prime}}^{-t}(\Omega)\right)^{\prime}(1<r \leq \infty), & -2 \leq t<0,\end{cases}
$$

where "cl" denotes the closure. Note that $u \in \mathbb{B}_{q, r}^{t}(\Omega)$ with $\frac{1}{q}<t \leq 2$ vanishes on $\partial \Omega$ by (5.1), but that only the normal component of $u$ vanishes on $\partial \Omega$ when $0<t \leq \frac{1}{q}$ since $u \in L_{\sigma}^{q}(\Omega)$. The definition for $t=0$ is more involved since the inclusion $L^{q}(\Omega) \subset B_{q, r}^{0}(\Omega)$ holds if and only if either $2 \leq q \leq r$ or $q \leq 2 \leq r$. For $r=\infty$, the space is also called Nikol'skii space and denoted by $\mathbb{N}_{q}^{t}(\Omega)$.

Moreover, we need the spaces (little Nikol'skii spaces, also denoted as $\mathbf{n}_{q}^{t}(\Omega)$ )

$$
\stackrel{\circ}{\mathbb{B}}_{q, \infty}^{t}(\Omega):=\operatorname{cl}\left(\mathbf{H}_{q}^{t}(\Omega) \cap L_{\sigma}^{q}(\Omega)\right) \text { in } \mathbb{B}_{q, \infty}^{t}(\Omega),
$$

where $\mathbf{H}_{q}^{t}(\Omega)$ is a Bessel potential space defined by restriction of the usual Bessel potential space $H_{q}^{t}\left(\mathbb{R}^{3}\right)^{3}$ to vector fields on $\Omega$ (vanishing on $\partial \Omega$ if $t>\frac{1}{q}$ as in (5.1)), cf. [3, pp. 3-4]. Using the notation $(\cdot, \cdot)_{\theta, r}, 1 \leq r<\infty$, of real interpolation, and $(\cdot, \cdot)_{\theta, \infty}^{0}$ for the continuous interpolation functor, Theorem 3.4 in [2] states that for $0<\theta<1$

$$
\begin{gathered}
\left(L_{\sigma}^{q}(\Omega), \mathcal{D}\left(A_{q}\right)\right)_{\theta, r}=\mathbb{B}_{q, r}^{2 \theta}(\Omega), \\
\left(L_{\sigma}^{q}(\Omega), \mathcal{D}\left(A_{q}\right)\right)_{\theta, \infty}^{0}=\mathbb{B}_{q, \infty}^{2 \theta}(\Omega) .
\end{gathered}
$$

Note that $\mathcal{D}\left(A_{q}\right)$ is equipped with its graph norm, and that for a bounded domain this graph norm can be simplified to $\left\|A_{q} \cdot\right\|_{q}$ since $0 \in \rho\left(A_{q}\right)$. As is well known ([23, Proposition 6.2, Exercise 6.1.1 (1)], equivalent norms on the spaces $\left(L_{\sigma}^{q}(\Omega), \mathcal{D}\left(A_{q}\right)\right)_{\theta, r}, 1 \leq r \leq \infty$, are given by

$$
\|u\|_{\mathbb{B}_{q, r}^{2 \theta}} \sim\left(\int_{0}^{T}\left(\tau^{1-\theta}\left\|A_{q} e^{-\tau A_{q}} u\right\|_{q}\right)^{r} \frac{\mathrm{d} \tau}{\tau}\right)^{1 / r} \text { if } 1 \leq r<\infty
$$

and for $r=\infty$

$$
\|u\|_{\left(L_{\sigma}^{q}, \mathcal{D}\left(A_{q}\right)\right)_{\theta, \infty}} \sim \sup _{(0, T)} \tau^{1-\theta}\left\|A_{q} e^{-\tau A_{q}} u\right\|_{q} \quad \text { if } r=\infty,
$$


where $T \in(0, \infty)$ can be chosen arbitrarily. The space $\stackrel{B}{B}_{q, \infty}^{2 \theta}(\Omega)$ is equipped with the norm of $\left(L_{\sigma}^{q}(\Omega), \mathcal{D}\left(A_{q}\right)\right)_{\theta, \infty}$, but elements $u \in \stackrel{\circ}{\mathbb{B}}_{q, \infty}^{2 \theta}(\Omega)$ enjoy the further property that

$$
\lim _{\tau \rightarrow 0} \tau^{1-\theta}\left\|A_{q} e^{-\tau A_{q}} u\right\|_{q}=0
$$

We note that the identity (5.4) for the usual interpolation space $\left(L_{\sigma}^{q}(\Omega), \mathcal{D}\left(A_{q}\right)\right)_{\theta, \infty}$ and the Besov space $\mathbb{B}_{q, \infty}^{2 \theta}(\Omega)$ is not found in the literature. This remark also applies to (5.12) dealing with negative exponents $\theta$.

To obtain similar representations for negative exponents of regularity as well recall that for $-1<\theta<0$ and $1<r \leq \infty$ by (5.2), (5.3) and the duality theorem of real interpolation ([30, Theorem 1.11.2])

$$
\left(L_{\sigma}^{q}(\Omega), \mathcal{D}\left(A_{q^{\prime}}\right)^{\prime}\right)_{-\theta, r}=\left(\left(L_{\sigma}^{q^{\prime}}(\Omega), \mathcal{D}\left(A_{q^{\prime}}\right)\right)_{-\theta, r^{\prime}}\right)^{\prime}=\left(\mathbb{B}_{q^{\prime}, r^{\prime}}^{-2 \theta}(\Omega)\right)^{\prime}=\mathbb{B}_{q, r}^{2 \theta}(\Omega) .
$$

Then we get the characterizations (here $-1<\theta<0$ ):

$$
\begin{aligned}
& \left(\mathcal{D}\left(A_{q^{\prime}}\right)^{\prime}, L_{\sigma}^{q}(\Omega)\right)_{1+\theta, r}=\mathbb{B}_{q, r}^{2 \theta}(\Omega), \quad 1 \leq r<\infty, \\
& \left(\mathcal{D}\left(A_{q^{\prime}}\right)^{\prime}, L_{\sigma}^{q}(\Omega)\right)_{1+\theta, \infty}=\mathbb{B}_{q, \infty}^{2 \theta}(\Omega) \cong \mathbf{B}_{q, \infty}^{2 \theta}(\Omega) /\left(\mathbb{B}_{q^{\prime}, 1}^{-2 \theta}(\Omega)\right)^{\perp}, \\
& \left(\mathcal{D}\left(A_{q^{\prime}}\right)^{\prime}, L_{\sigma}^{q}(\Omega)\right)_{1+\theta, \infty}^{0}=\mathbb{B}_{q, \infty}^{2 \theta}(\Omega)=\operatorname{cl}\left(\mathbf{H}_{q}^{2}(\Omega)\right) \text { in }\left(\mathbb{B}_{q^{\prime}, 1}^{-2 \theta}(\Omega)\right)^{\prime} .
\end{aligned}
$$

Actually, (5.8) for $r=1$ and (5.10) follow from [2, Theorem 3.4], [3, p. 4], for all $-1<\theta<0$; the space $\stackrel{\circ}{B}_{q, \infty}^{2 \theta}(\Omega)$ also coincides with the closure $\operatorname{cl}\left(L_{\sigma}^{q}(\Omega)\right)$ in $\mathbb{B}_{q, \infty}^{2 \theta}(\Omega)$. To prove (5.9), we exploit the isomorphism

$$
\mathbb{B}_{q, \infty}^{2 \theta}(\Omega)=\left(\mathbb{B}_{q^{\prime}, 1}^{-2 \theta}(\Omega)\right)^{\prime} \cong \mathbf{B}_{q, \infty}^{2 \theta}(\Omega) /\left(\mathbb{B}_{q^{\prime}, 1}^{-2 \theta}(\Omega)\right)^{\perp}
$$

see [2, Remark 3.6] and its proof as well as definitions (5.1), (5.2).

Furthermore, note from Sect. 2 that $A_{q}$ is an isomorphism from $\mathcal{D}\left(A_{q}\right)$ to $L_{\sigma}^{q}(\Omega)$ and also from $L_{\sigma}^{q}(\Omega)$ to $\mathcal{D}\left(A_{q^{\prime}}\right)^{\prime}$. Hence, for all $1 \leq r \leq \infty$ and $-1<\theta<0$

$$
\left(\mathcal{D}\left(A_{q^{\prime}}\right)^{\prime}, L_{\sigma}^{q}(\Omega)\right)_{1+\theta, r}=A\left(\left(L_{\sigma}^{q}(\Omega), \mathcal{D}\left(A_{q}\right)\right)_{1+\theta, r}\right),
$$

with a similar result for the continuous interpolation functor $(\cdot, \cdot)_{\theta, \infty}^{0}$.

Thus, for any $1 \leq r \leq \infty$ and $-1<\theta<0$, by (5.11), (5.8), (5.9) and (5.3), $\left(\mathcal{D}\left(A_{q^{\prime}}\right)^{\prime}, L_{\sigma}^{q}(\Omega)\right)_{1+\theta, r}=A\left(\left(L_{\sigma}^{q}(\Omega), \mathcal{D}\left(A_{q}\right)\right)_{1+\theta, r}\right)=\mathbb{B}_{q, r}^{2 \theta}(\Omega)$ with equivalent norm

$$
\|u\|_{A\left(\left(L_{\sigma}^{q}(\Omega), \mathcal{D}\left(A_{q}\right)\right)_{1+\theta, r}\right)} \sim \begin{cases}\left(\int_{0}^{T}\left(\tau^{-\theta}\left\|e^{-\tau A_{q}} u\right\|_{q}\right)^{r} \frac{\mathrm{d} \tau}{\tau}\right)^{1 / r} & \text { if } 1 \leq r<\infty, \\ \sup _{\tau \in(0, T)} \tau^{-\theta}\left\|e^{-\tau A_{q}} u\right\|_{q} & \text { if } r=\infty .\end{cases}
$$

This result was used in [12] when $\frac{2}{r}+\frac{3}{q}=1, \theta=0,2<r<\infty$. For the continuous interpolation space $\left(\mathcal{D}\left(A_{q^{\prime}}\right)^{\prime}, L_{\sigma}^{q}(\Omega)\right)_{1+\theta, \infty}^{0}=\stackrel{\circ}{\mathbb{B}}_{q, \infty}^{2 \theta}(\Omega)$, we have the norm defined in (5.12), with the additional property that

$$
\lim _{\tau \rightarrow 0} \tau^{-\theta}\left\|e^{-\tau A_{q}} u\right\|_{q}=0 .
$$

Summarizing the previous arguments, we get the following theorem. 
Theorem 5.1 Choose any $T \in(0, \infty)$.

(i) Let $2<s<\infty, 3<q<\infty$ and $0<\alpha<\frac{1}{2}$ such that $\frac{2}{s}+\frac{3}{q}=1-2 \alpha$. Then the real interpolation space $\left(\mathcal{D}\left(A_{q^{\prime}}\right)^{\prime}, L_{\sigma}^{q}(\Omega)\right)_{1-\alpha, s}$ coincides with the Besov space $\mathbb{B}_{q, s}^{-1+3 / q}(\Omega)$ and has the equivalent norm $\left(\int_{0}^{T}\left(\tau^{\alpha}\left\|e^{-\tau A_{q}} u\right\|_{q}\right)^{s} \mathrm{~d} \tau\right)^{1 / s}$.

(ii) If $3<q<\infty$ and $0<\alpha<\frac{1}{2}$ such that $\frac{3}{q}=1-2 \alpha$, the real interpolation space $\left(\mathcal{D}\left(A_{q^{\prime}}\right)^{\prime}, L_{\sigma}^{q}(\Omega)\right)_{1-\alpha, \infty}$ coincides with the space of Besov type $\mathbb{B}_{q, \infty}^{-1+3 / q}(\Omega)$ and has the equivalent norm $\sup _{\tau \in(0, T)} \tau^{\alpha}\left\|e^{-\tau A_{q}} u\right\|_{q}$.

(iii) The interpolation space $\left(\mathcal{D}\left(A_{q^{\prime}}\right)^{\prime}, L_{\sigma}^{q}(\Omega)\right)_{1-\alpha, \infty}^{0}$ equals the Besov space $\stackrel{\circ}{\mathbb{B}}_{q, \infty}^{-1+3 / q}(\Omega)$, equipped with the norm of $\mathbb{B}_{q, \infty}^{-1+3 / q}(\Omega)$ such that the property $\lim _{\tau \rightarrow 0} \tau^{\alpha}\left\|e^{-\tau A_{q}} u\right\|_{q}=0$ additionally holds for $u \in \mathbb{B}_{q, \infty}^{-1+3 / q}(\Omega)$.

Acknowledgements This work is partly supported by the Japan Society for the Promotion of Science (JSPS) and the German Research Foundation through Japanese-German Graduate Externship IRTG 1529. The first and third authors are supported in part by the 7th European Framework Programme IRSES "FLUX", Grant Agreement No. PIRSES-GA-2012-319012. The second author is partly supported by JSPS through the Grant Kiban S (26220702), Kiban A (17H01091), Kiban B (16H03948), Challenging Research (Pioneering) (18H05323). Moreover, the third author gratefully acknowledges the support by the Iwanami Fujukai Foundation. We also would like to thank the anonymous reviewer for helpful comments to improve the style of this article.

\section{References}

1. Amann, H.: Linear and Quasilinear Parabolic Equations. Birkhäuser Verlag, Basel (1995)

2. Amann, H.: On the strong solvability of the Navier-Stokes equations. J. Math. Fluid Mech. 2, 16-98 (2000)

3. Amann, H.: Navier-Stokes equations with nonhomogeneous Dirichlet data. J. Nonlinear Math. Phys. 10(Suppl. 1), 1-11 (2003)

4. Bourgain, J., Pavlović, N.: Ill-posedness of the Navier-Stokes equations in a critical space in 3D. J. Funct. Anal. 255, 2233-2247 (2008)

5. Cheskidov, A., Shvydkoy, R.: Ill-posedness of the basic equations of fluid dynamics in Besov spaces. Proc. Am. Math. Soc. 138, 1059-1067 (2010)

6. Engel, K.-J., Nagel, R.: One-Parameter Semigroups for Linear Evolution Equations. Springer, Berlin (2000)

7. Farwig, R.: On regularity of weak solutions to the instationary Navier-Stokes system: a review on recent results. Ann. Univ. Ferrara, Sez. VII. Sci. Mat. 60, 91-122 (2014)

8. Farwig, R., Giga, Y., Hsu, P.-Y.: Initial values for the Navier-Stokes equations in spaces with weights in time. Funkcial. Ekvac. 59, 199-216 (2016)

9. Farwig, R., Giga, Y., Hsu, P.-Y.: The Navier-Stokes equations with initial values in Besov spaces of type $B_{q, \infty}^{-1+3 / q}$. J. Korean Math. Soc. 54, 1483-1504 (2017)

10. Farwig, R., Sohr, H.: Generalized resolvent estimates for the Stokes system in bounded and unbounded domains. J. Math. Soc. Japan 46, 607-643 (1994)

11. Farwig, R., Sohr, H.: Optimal initial value conditions for the existence of local strong solutions of the Navier-Stokes equations. Math. Ann. 345, 631-642 (2009)

12. Farwig, R., Sohr, H., Varnhorn, W.: On optimal initial value conditions for local strong solutions of the Navier-Stokes equations. Ann. Univ. Ferrara Sez. VII. Sci. Mat. 55, 89-110 (2009)

13. Fujita, H., Kato, T.: On the Navier-Stokes initial value problem. Arch. Ration. Mech. Anal. 16, 269-315 (1964)

14. Giga, Y.: Solution for semilinear parabolic equations in $L^{p}$ and regularity of weak solutions for the Navier-Stokes system. J. Differ. Equ. 61, 186-212 (1986)

15. Giga, Y., Miyakawa, T.: Solutions in $L_{r}$ of the Navier-Stokes initial value problem. Arch. Ration. Mech. Anal. 89, 267-281 (1985)

16. Haak, B.H., Kunstmann, P.C.: On Kato's method for Navier-Stokes equations. J. Math. Fluid Mech. 11, 492-535 (2009) 
17. Heywood, J.G.: The Navier-Stokes equations: on the existence, regularity and decay of solutions. Indiana Univ. Math. J. 29, 639-681 (1980)

18. Kato, T.: Strong $L^{p}$-solutions of the Navier-Stokes equation in $\mathbb{R}^{m}$, with applications to weak solutions. Math. Z. 187, 471-480 (1984)

19. Kiselev, A.A., Ladyzhenskaya, O.A.: On the existence and uniqueness of solutions of the non-stationary problems for flows of non-compressible fluids. Am. Math. Soc. Transl. II 24, 79-106 (1963)

20. Koch, H., Tataru, D.: Well-posedness for the Navier-Stokes equations. Adv. Math. 157, 22-35 (2001)

21. Kozono, H., Yamazaki, M.: Local and global unique solvability of the Navier-Stokes exterior problem with Cauchy data in the space $L^{n, \infty}$. Houst. J. Math. 21, 755-799 (1995)

22. Leray, J.: Sur le mouvement d'un liquide visqueux emplissant l'espace. Acta Math. 63, 193-248 (1934)

23. Lunardi, A.: Interpolation Theory. Edizioni Della Normale, Appunti. Sc. Norm. Super. Pisa, 3rd edn. (2018)

24. Meyer, Y.: Wavelets, Paraproducts and Navier-Stokes Equations. Current Developments in Mathematics. International Press, Boston (1997)

25. Miyakawa, T.: On the initial value problem for the Navier-Stokes equations in $L^{r}$-spaces. Hiroshima Math. J. 11, 9-20 (1981)

26. Sohr, H.: The Navier-Stokes Equations. An Elementary Functional Analytic Approach. Birkhäuser, Basel (2001)

27. Solonnikov, V.A.: Estimates for solutions of nonstationary Navier-Stokes equations. J. Sov. Math. 8, 467-529 (1977)

28. Stein, E.M., Weiss, G.: Fractional integrals on n-dimensional Euclidean space. J. Math. Mech. 7, 503-514 (1958)

29. Strichartz, R.S.: $L^{p}$ estimates for integral transforms. Trans. Am. Math. Soc. 136, 33-50 (1969)

30. Triebel, H.: Interpolation Theory, Function Spaces. Differential Operators, Amsterdam (1978)

31. Wang, B.: Ill-posedness for the Navier-Stokes equations in critical Besov spaces $\dot{B}_{\infty, q}^{-1}$. Adv. Math. 268, 350-372 (2015)

32. Yoneda, T.: Ill-posedness of the 3D-Navier-Stokes equations in a generalized Besov space near $B M O^{-1}$. J. Funct. Anal. 258, 3376-3387 (2010)

Publisher's Note Springer Nature remains neutral with regard to jurisdictional claims in published maps and institutional affiliations. 